\title{
Posterior mediastinal mass compressing the left atrium: A rare complication after lung resection
}

\author{
Naase $H^{*}$, Rescigno G, Williams J, Rathod J and Kornaszewska M \\ Department of Cardiothoracic Surgery, University Hospital of Wales, Heath Park Cardiff, UK
}

\begin{abstract}
We are presenting a rare postoperative lung resection complication, which led to secondary constraints on the left sided heart, leading to pure cardiac decompensation with recovered pulmonary function. This complication comprises of a large contained mediastinal haematoma with severe left atrial and oesophageal compression.
\end{abstract}

\section{Background}

It is not uncommon that the postoperative course of any pulmonary resection may be complicated by dyspnoea. The severity of this symptom is multifactorial; it varies according to the preoperative performance status, the nature of resected tissue, degree of postoperative pain and additionally, the post-operative complications such as pneumothorax or retained secretions and lung collapse. Other possible aetiologies are cardiac related (i.e. left or right ventricle dysfunction, preexisting valvulopathies). Here, we describe a progressively increasing postoperative dyspnoea that was produced by a posterior mediastinal mass compressing the left atrium.

\section{Case report}

A 71-year-old woman with COPD and current smoker was referred to our department for a left upper lobe lesion with avid $3.2 \mathrm{~cm}$ mass on Positron Emission Tomography (PET) scan. No nodal or distant metastatic disease and staged as T2aN0M0. A computed tomography (CT) guided core biopsies showed foci of moderately differentiated squamous cell carcinoma. A surgical resection was performed in form of uniportal left VATS and upper lobectomy with enblock excision of mediastinal lymph nodes including the aorto-pulmonary (AP) window (station 5), subcarinal (station 7), hilar (station 10) and inter-lobar (station 11) lymph nodes. The subcarinal lymph node dissection was done utilizing the anterior approach. The subsequent histopathology later confirms the tumour to be a moderately differentiated squamous cell carcinoma and was completely excised. The stations 5,7,10 and 11 were reactive lymph nodes and free from tumour. There were no intraoperative or immediate postoperative complications. The patient was extubated in the operating room and chest tube was removed on the second postoperative day with no significant fluid drainage or air leak. The patient developed atrial fibrillation on the second postoperative day, which was successfully pharmacologically cardioverted without the need to use anticoagulation. Despite this apparent uneventful recovery, the patient experienced significant shortness of breath and hoarseness of voice with essentially normal chest radiogram. On the fifth postoperative day, an evident lower limb swelling was observed. A transthoracic echocardiogram was performed showing a normal biventricular function with normal functioning cardiac valves. However, there was a large mediastinal mass severely compressing the posterior wall of the left atrium (Figure 1). A subsequent CT scan demonstrated a well circumscribed posterior mediastinal mass, located anterior to the aorta and extending toward the right side. Its largest diameter was $5.8 \mathrm{~cm}$. The oesophagus was significantly displaced to the left side (Figure 2); there was no active bleeding as demonstrated by the absence of contrast medium. This mass was definitely related to surgery, as it was not present in the preoperative scans. It was believed that the mass is a haematoma related to the lymph node dissected, therefore, the patient was re-operated on using the same uniportal incision, an organised haematoma was found at the site of the subcarinal lymph node dissection, which was done previously through the anterior hilar approach (Figure 3). It was compressing both the left atrium and oesophagus and was adjacent to the main pulmonary artery (PA). The haematoma then was evacuated and dissected from the main PA with no subsequent bleeding observed after multiple washouts with warm $0.9 \%$ Saline. The subsequent postoperative course was uneventful; with complete resolution of dyspnoea, hoarseness of voice and pedal oedema. The pre-discharge echocardiogram and CT angiogram demonstrated complete removal of the mass and a normal left atrial cavity.

\section{Discussion}

It is known that lung resection leads to a reduction in lung function proportionally to the number of segments resected [1]. Moreover, the symptoms in most of the cases are related to the obvious loss of lung volume leading to inadequate gas exchange. This condition usually improves with time aided by pulmonary rehabilitation and physiotherapy. Other causes are retained secretions, pneumothorax or pleural effusion especially after drain removal. However, the posterior significant mediastinal haematomas after lung resection are uncommon

Correspondence to: Hatam Naase, Department of Cardiothoracic Surgery, University Hospital of Wales, Heath Park Cardiff, UK; E-mail: hatamnaase@ doctors.org.uk

Key words: lung resection, postoperative pain, squamous cell carcinoma, haematomas

Received: November 08, 2017; Accepted: November 28, 2017; Published: December 02, 2017 


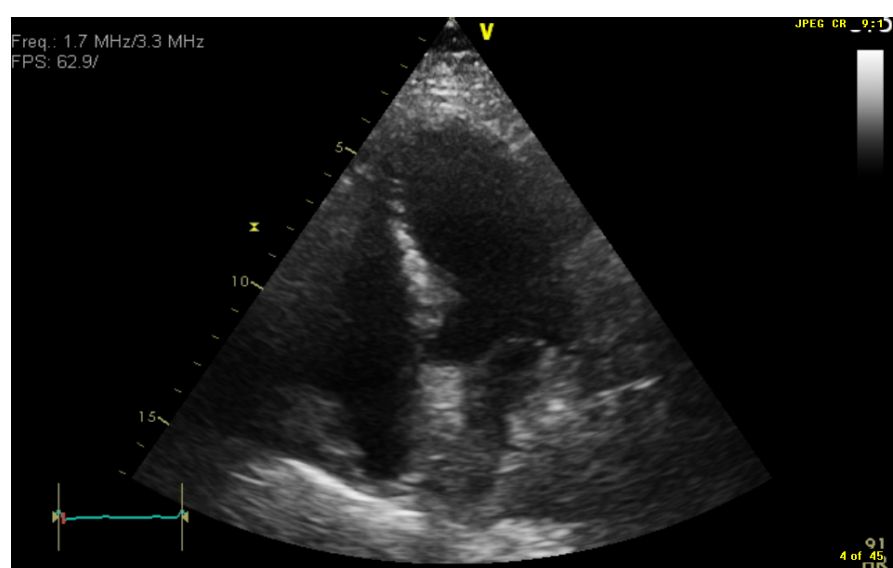

Figure 1. Trans-thoracic echocardiogram showing compression of the left atrium from the posterior mediastinal mass.

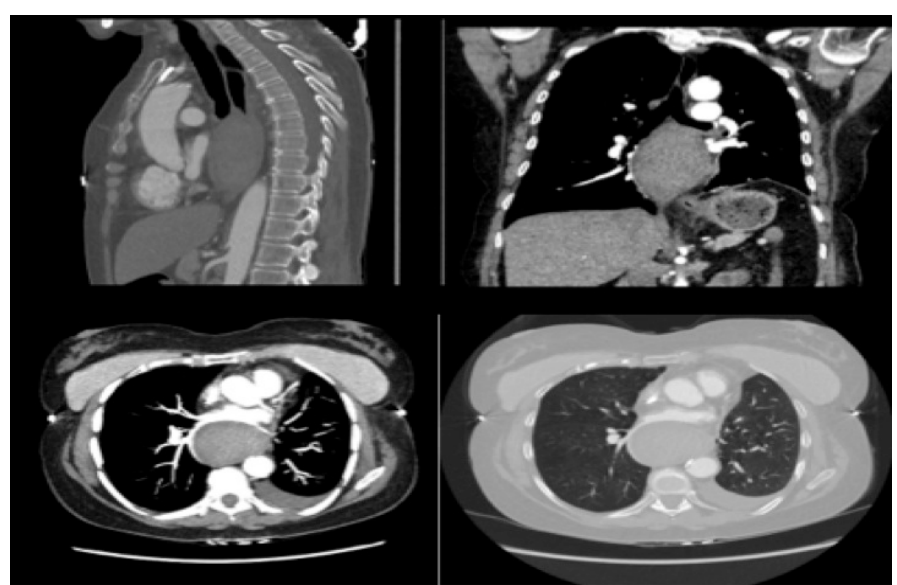

Figure 2. CT scan demonstrating the posterior mediastinal mass compression on the adjacent structure.

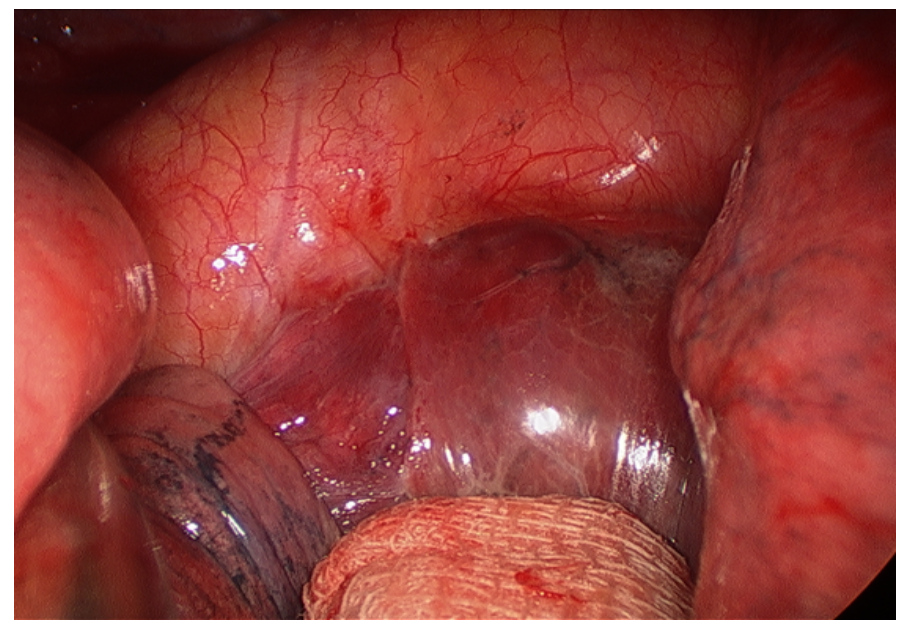

Figure 3. Intra-operative view of the contained posterior mediastinal haematoma

and this is a rare location which is not highlighted in the literatures, in contrast, few cases of anterior mediastinal haematoma have been reported after cardiac surgery with compression of the right atrium or the left ventricle with outflow tract obstruction [2-4]. The most frequent reported haematoma are those related to the thoracic trauma [5]. However, spontaneous haematomas are rare but can occur due to anticoagulation usage $[6,7]$ or related to bleeding from mediastainal structures due to sudden increase in intrathoracic pressure such as in vomiting, sneezing or coughing [8]. In the present case, this is the first to describe a postoperative haematoma after lung surgery. The cause of this well contained blood collection is probably related to mediastinal lymphadenectomy of the subcarinal or AP window lymph nodes (Station 5 and 7) in which here we have used the anterior approach as opposed to the usual posterior approach due to adhesions.

This unusual finding was considered when all pulmonary causes had been excluded. In the presence of new bilateral lower-limb oedema the differential diagnosis of symptoms being secondary to a cardiac cause was investigated. In fact, the findings on the transthoracic echocardiogram were unexpected. The clinical condition was produced by left atrial compression by a large collection of clots causing symptoms due to left atrial overload leading to pulmonary congestion. Its evacuation led to a rapid improvement of the patient condition and subsequent uneventful recovery.

\section{Conclusion}

In conclusion, we believe that this present case demonstrates the value and importance of careful analysis of any postoperative complications to facilitate prompt and proper management. This illustrate that unexplained symptoms may be indicative for underlying serious cause, this should prompt further investigation and definitive action in which here was immediate surgical intervention. This enables full evaluation of the patient to avoid missed serious complications.

\section{References}

1. Cukkic V (2012) Preoperative prediction of lung function in pneumonectomy by spirometry and lung perfusion scintigraphy. Acta Inform Med 20: 221-225. [Crossref]

2. Jordan AJ, Garcia M, Santos F, Gonzalez M, Gomez J, et al. (2001) Shock secondary to extrinsic compression of the right atrium by postoperative mediastinal hematoma Pseudotumor echocardiography image in right atrium. Rev Esp Cardiol 54: 396-398. [Crossref]

3. Alsafwah S, Minderman D, Mallisho M, Munir A (2008) Mediastinal hematoma causing compression of the right ventricular outflow tract--the role of transthoracic echocardiography in diagnosis. Can J Cardiol 24: 644. [Crossref]

4. Tardif JC, Taylor K, Pandian NG, Schwartz S, Rastegar H (1994) Right ventricular outflow tract and pulmonary artery obstruction by postoperative mediastinal hematoma: delineation by multiplane transesophageal echocardiography. J Am Soc Echocardiogr 7: 400-404. [Crossref]

5. Rojas CA, Restrepo CS (2009) Mediastinal hematomas: aortic injury and beyond. $J$ Comput Assist Tomogr 33: 218-224. [Crossref]

6. Mikubo M, Sonoda D, Yamazaki H, Naito M, Matsui Y, et al. (2017) Spontaneous nontraumatic mediastinal hematoma associated with oral anticoagulant therapy: A case report and literature review. Int J Surg Case Rep 39: 221-224. [Crossref]

7. Jette LA, Niven AS, Nieves-Robbins N (2011) Spontaneous mediastinal haematoma: a rare complication of warfarin therapy. BMJ Case Rep 2011. [Crossref]

8. Restrepo CS, Lemos DF, Ocazionez D, Moncada R, Gimenez CR (2008) Intramural hematoma of the esophagus: a pictorial essay. Emerg Radiol 15: 13-22. [Crossref]

Copyright: (C)2017 Naase H. This is an open-access article distributed under the terms of the Creative Commons Attribution License, which permits unrestricted use, distribution, and reproduction in any medium, provided the original author and source are credited. 